\title{
BOHR RADIUS FOR LOCALLY UNIVALENT HARMONIC MAPPINGS
}

\author{
ILGIZ R KAYUMOV, SAMINATHAN PONNUSAMY, NAIL SHAKIROV
}

\begin{abstract}
We consider the class of all sense-preserving harmonic mappings $f=h+\bar{g}$ of the unit disk $\mathbb{D}$, where $h$ and $g$ are analytic with $g(0)=0$, and determine the Bohr radius if any one of the following conditions holds:

(1) $h$ is bounded in $\mathbb{D}$.

(2) $h$ satisfies the condition $\operatorname{Re} h(z) \leq 1$ in $\mathbb{D}$ with $h(0)>0$.

(3) both $h$ and $g$ are bounded in $\mathbb{D}$.

(4) $h$ is bounded and $g^{\prime}(0)=0$.

We also consider the problem of determining the Bohr radius when the supremum of the modulus of the dilatation of $f$ in $\mathbb{D}$ is strictly less than 1 . In addition, we determine the Bohr radius for the space $\mathcal{B}$ of analytic Bloch functions and the space $\mathcal{B}_{H}$ of harmonic Bloch functions. The paper concludes with two conjectures.
\end{abstract}

\section{Introduction AND PRELiminaries}

We shall investigate Bohr's radius for complex-valued harmonic mappings and locally univalent harmonic mappings defined on the unit disk $\mathbb{D}:=\{z \in \mathbb{C}:|z|<1\}$. The Bohr theorem about power series (after subsequent improvements due to M. Riesz, I. Schur and F. Wiener) states that if $f$ is a bounded analytic function on $\mathbb{D}$, with the Taylor expansion $f(z)=\sum_{n=0}^{\infty} a_{n} z^{n}$, then

$$
\sum_{n=0}^{\infty}\left|a_{n}\right| r^{n} \leq\|f\|_{\infty}
$$

for $0<r \leq 1 / 3$ and the constant $1 / 3$ is sharp. The best constant $r$ in the above inequality, which is $1 / 3$, is called the Bohr radius for the class of all analytic self-maps of the unit disk $\mathbb{D}$. The original problem goes back to 1914's. Many mathematicians have contributed toward the understanding of this problem in several settings. We refer to the recent survey on this topic by Abu-Muhanna et al. [1] for the importance, background, and several other recent results and extensions. For certain recent results, see [2,11,12].

A harmonic mapping in $\mathbb{D}$ is a complex-valued function $f=u+i v$ of $z=x+i y$ in $\mathbb{D}$, which satisfies the Laplace equation $\Delta f=4 f_{z \bar{z}}=0$, where $f_{z}=(1 / 2)\left(f_{x}-i f_{y}\right)$ and $f_{\bar{z}}=(1 / 2)\left(f_{x}+i f_{y}\right)$ and where $u$ and $v$ are real-valued harmonic functions on $\mathbb{D}$. It follows that $f$ admits the canonical representation $f=h+\bar{g}$, where $h$ and $g$ are analytic in $\mathbb{D}$ with $f(0)=h(0)$. The Jacobian $J_{f}$ of $f$ is given by $J_{f}=\left|h^{\prime}\right|^{2}-\left|g^{\prime}\right|^{2}$. We say that $f$ is sense-preserving in $\mathbb{D}$ if $J_{f}(z)>0$ in $\mathbb{D}$. Consequently, $f$ is locally univalent and

2000 Mathematics Subject Classification. Primary: 30A10, 30B10, 30C62, 31A05; Secondary: 30C75,

Key words and phrases. Harmonic, locally univalent, and analytic functions, Schwarz Lemma, Bloch space, Bohr radius, $K$-Quasiconformal mappings.

File: KayShak4'BohrQC'final.tex, printed: 15-9-2017, 0.23. 
sense-preserving in $\mathbb{D}$ if and only if $J_{f}(z)>0$ in $\mathbb{D}$; or equivalently if $h^{\prime} \neq 0$ in $\mathbb{D}$ and the dilatation $\omega_{f}=: \omega=g^{\prime} / h^{\prime}$ has the property that $|\omega(z)|<1$ in $\mathbb{D}$ (see [14]).

In order to state the first result about Bohr radius for quasiconformal harmonic mappings, we need to introduce some notation. For harmonic mappings $f$ in $\mathbb{D}$, we use the following standard notations:

$$
\Lambda_{f}(z)=\max _{0 \leq \theta \leq 2 \pi}\left|f_{z}(z)+e^{-2 i \theta} f_{\bar{z}}(z)\right|=\left|f_{z}(z)\right|+\left|f_{\bar{z}}(z)\right|
$$

and

$$
\lambda_{f}(z)=\min _{0 \leq \theta \leq 2 \pi}\left|f_{z}(z)+e^{-2 i \theta} f_{\bar{z}}(z)\right|=|| f_{z}(z)|-| f_{\bar{z}}(z)||
$$

so that if $f$ is locally univalent and sense-preserving, then

$$
J_{f}=\lambda_{f} \Lambda_{f}=\left|f_{z}\right|^{2}-\left|f_{\bar{z}}\right|^{2}>0 .
$$

A sense-preserving homeomorphism $f$ from the unit disk $\mathbb{D}$ onto $\Omega^{\prime}$, contained in the Sobolev class $W_{l o c}^{1,2}(\mathbb{D})$, is said to be a $K$-quasiconformal mapping if, for $z \in \mathbb{D}$,

$$
\frac{\left|f_{z}\right|+\left|f_{\bar{z}}\right|}{\left|f_{z}\right|-\left|f_{\bar{z}}\right|}=\frac{1+\left|\omega_{f}(z)\right|}{1-\left|\omega_{f}(z)\right|} \leq K, \text { i.e., }\left|\omega_{f}(z)\right| \leq k=\frac{K-1}{K+1}
$$

where $K \geq 1$ so that $k \in[0,1)$ (cf. [13, 16]).

Theorem 1. Suppose that $f(z)=h(z)+\overline{g(z)}=\sum_{n=0}^{\infty} a_{n} z^{n}+\overline{\sum_{n=1}^{\infty} b_{n} z^{n}}$ is a sensepreserving $K$-quasiconformal harmonic mapping of the disk $\mathbb{D}$, where $h$ is a bounded function in $\mathbb{D}$. Then

$$
\sum_{n=0}^{\infty}\left|a_{n}\right| r^{n}+\sum_{n=1}^{\infty}\left|b_{n}\right| r^{n} \leq\|h\|_{\infty} \text { for } r \leq \frac{K+1}{5 K+1} .
$$

The constant $(K+1) /(5 K+1)$ is sharp.

Theorem 2. Assume the hypothesis of Theorem 1. Then

$$
\left|a_{0}\right|^{2}+\sum_{n=1}^{\infty}\left(\left|a_{n}\right|+\left|b_{n}\right|\right) r^{n} \leq\|h\|_{\infty} \text { for } r \leq \frac{K+1}{3 K+1} .
$$

The constant $(K+1) /(3 K+1)$ is sharp.

We would like to remark that the boundedness condition on $h$ in Theorem 1 can be replaced by half-plane condition. However, the Bohr radius remains the same in this case too.

Theorem 3. Suppose that $f(z)=h(z)+\overline{g(z)}=\sum_{n=0}^{\infty} a_{n} z^{n}+\overline{\sum_{n=1}^{\infty} b_{n} z^{n}}$ is a sensepreserving $K$-quasiconformal harmonic mapping of the disk $\mathbb{D}$, where $h$ satisfies the condition $\operatorname{Re} h(z) \leq 1$ in $\mathbb{D}$ and $h(0)=a_{0}$ is positive. Then

$$
a_{0}+\sum_{n=1}^{\infty}\left|a_{n}\right| r^{n}+\sum_{n=1}^{\infty}\left|b_{n}\right| r^{n} \leq 1 \text { for } r \leq \frac{K+1}{5 K+1} .
$$

The constant $(K+1) /(5 K+1)$ is sharp. 
The following corollaries are regarded as harmonic analogs of the classical Bohr inequality and will be of independent interest. These results are obtained by allowing $K \rightarrow \infty$ in the above results.

Corollary 1. Suppose that $f(z)=h(z)+\overline{g(z)}=\sum_{n=0}^{\infty} a_{n} z^{n}+\overline{\sum_{n=1}^{\infty} b_{n} z^{n}}$ is a sensepreserving harmonic mapping of the disk $\mathbb{D}$, where $h$ is a bounded function in $\mathbb{D}$. Then

$$
\left|a_{0}\right|+\sum_{n=1}^{\infty}\left(\left|a_{n}\right|+\left|b_{n}\right|\right) r^{n} \leq\|h\|_{\infty} \text { for } r \leq \frac{1}{5},
$$

and the number $1 / 5$ is sharp. Moreover, either $a_{0}=0$ or $\left|a_{0}\right|$ in (1) is replaced by $\left|a_{0}\right|^{2}$, then the constant $1 / 5$ could be replaced by $1 / 3$ which is also sharp.

Corollary 2. Suppose that $f(z)=h(z)+\overline{g(z)}=\sum_{n=0}^{\infty} a_{n} z^{n}+\overline{\sum_{n=1}^{\infty} b_{n} z^{n}}$ is a sensepreserving harmonic mapping of the disk $\mathbb{D}$, where $h$ satisfies the condition $\operatorname{Re} h(z) \leq 1$ in $\mathbb{D}$ and $h(0)=a_{0}$ is positive. Then

$$
a_{0}+\sum_{n=1}^{\infty}\left(\left|a_{n}\right|+\left|b_{n}\right|\right) r^{n} \leq 1 \text { for } r \leq \frac{1}{5}
$$

and the number $1 / 5$ is sharp.

Theorem 4. Suppose that either $f=h+g$ or $f=h+\bar{g}$, where $h(z)=\sum_{n=1}^{\infty} a_{n} z^{n}$ and $g(z)=\sum_{n=1}^{\infty} b_{n} z^{n}$ are bounded analytic functions in $\mathbb{D}$. Then

$$
\sum_{n=1}^{\infty}\left(\left|a_{n}\right|+\left|b_{n}\right|\right) r^{n} \leq \max \left\{\|h\|_{\infty},\|g\|_{\infty}\right\} \text { for } r \leq \sqrt{\frac{7}{32}}
$$

This number $\sqrt{7 / 32}$ is sharp.

As in the symmetric case of analytic functions (see [2, 11, 12]), we have the following analog result for harmonic functions.

Theorem 5. Let $p \geq 2$. Suppose that $f(z)=h(z)+\overline{g(z)}=\sum_{n=0}^{\infty} a_{n} z^{p n+1}+\overline{\sum_{n=0}^{\infty} b_{n} z^{p n+1}}$ is a harmonic p-symmetric function in $\mathbb{D}$, where $h$ and $g$ are bounded functions in $\mathbb{D}$. Then

$$
\sum_{n=0}^{\infty}\left(\left|a_{n}\right|+\left|b_{n}\right|\right) r^{p n+1} \leq \max \left\{\|h\|_{\infty},\|g\|_{\infty}\right\} \text { for } r \leq \frac{1}{2}
$$

The number $1 / 2$ is sharp.

The proofs of these results will be given in Section 2. In Section 3, we extend further results for sense-preserving $K$-quasiconformal harmonic mappings of the disk $\mathbb{D}$. In Section 4, we consider the problem of finding the Bohr radius for the space of bounded harmonic Bloch functions. The paper concludes with a couple of conjectures.

\section{The Proofs of Theorems 1, 2, 3, 4] and 5}

The following lemma is needed for the proof our first theorem. 
Lemma 1. Suppose that $h(z)=\sum_{n=0}^{\infty} a_{n} z^{n}$ and $g(z)=\sum_{n=0}^{\infty} b_{n} z^{n}$ are two analytic functions in the unit disk $\mathbb{D}$ such that $\left|g^{\prime}(z)\right| \leq k\left|h^{\prime}(z)\right|$ in $\mathbb{D}$ and for some $k \in[0,1]$. Then

$$
\sum_{n=1}^{\infty}\left|b_{n}\right|^{2} r^{n} \leq k^{2} \sum_{n=1}^{\infty}\left|a_{n}\right|^{2} r^{n} \quad \text { for } \quad|z|=r<1 .
$$

Proof. We integrate inequality $\left|g^{\prime}(z)\right|^{2} \leq k^{2}\left|h^{\prime}(z)\right|^{2}$ over the circle $|z|=r$ and get

$$
\sum_{n=1}^{\infty} n^{2}\left|b_{n}\right|^{2} r^{2(n-1)} \leq k^{2} \sum_{n=1}^{\infty} n^{2}\left|a_{n}\right|^{2} r^{2(n-1)} .
$$

We integrate the last inequality with respect to $r^{2}$ and obtain

$$
\sum_{n=1}^{\infty} n\left|b_{n}\right|^{2} r^{2 n} \leq k^{2} \sum_{n=1}^{\infty} n\left|a_{n}\right|^{2} r^{2 n}
$$

One more integration (after dividing by $r^{2}$ ) gives desired inequality.

Proof of Theorem 1. For simplicity, we suppose that $\|h\|_{\infty}=1$. Then $\left|a_{n}\right| \leq 1-\left|a_{0}\right|^{2}$ for $n \geq 1$. Let $\omega$ denote the dilatation of $f=h+\bar{g}$ so that $\left|g^{\prime}(z)\right| \leq k\left|h^{\prime}(z)\right|$ in $\mathbb{D}$, where $k \in[0,1)$ and so, by Lemma 1, it follows that

$$
\sum_{n=1}^{\infty}\left|b_{n}\right|^{2} r^{n} \leq k^{2} \sum_{n=1}^{\infty}\left|a_{n}\right|^{2} r^{n} \leq k^{2}\left(1-\left|a_{0}\right|^{2}\right)^{2} \frac{r}{1-r} .
$$

Consequently,

$$
\sum_{n=1}^{\infty}\left|b_{n}\right| r^{n} \leq \sqrt{\sum_{n=1}^{\infty}\left|b_{n}\right|^{2} r^{n}} \sqrt{\sum_{n=1}^{\infty} r^{n}} \leq k\left(1-\left|a_{0}\right|^{2}\right) \frac{r}{1-r}
$$

so that

$$
\sum_{n=0}^{\infty}\left|a_{n}\right| r^{n}+\sum_{n=1}^{\infty}\left|b_{n}\right| r^{n} \leq\left|a_{0}\right|+\left(1-\left|a_{0}\right|^{2}\right)(1+k) \frac{r}{1-r}
$$

which is clearly less than or equal to 1 for $r \leq 1 /(3+2 k)$. Substituting $k=(K-1) /(K+1)$ gives the desired result.

To prove the sharpness, consider

$$
h(z)=\frac{a-z}{1-\bar{a} z}=a+\sum_{n=1}^{\infty} a_{n} z^{n}, \quad a_{n}=-\left(1-|a|^{2}\right)(\bar{a})^{n-1} \text { for } n \geq 1,
$$

and $g(z)=\lambda k h(z)$, where $|\lambda|=1, a \in \mathbb{D}$ and $k=(K-1) /(K+1)$. Then it is a simple exercise to see that

$$
\sum_{n=0}^{\infty}\left|a_{n}\right| r^{n}+\sum_{n=1}^{\infty}\left|b_{n}\right| r^{n}=|a|+\left(1-|a|^{2}\right)(1+k) \sum_{n=1}^{\infty}|a|^{n-1} r^{n}=|a|+\left(1-|a|^{2}\right)(1+k) \frac{r}{1-|a| r}
$$

which is bigger than or equal to 1 if and only if

$$
r \geq \frac{1}{1+k+(2+k)|a|}=\frac{K+1}{2 K+(3 K+1)|a|} .
$$


This shows that the number $(K+1) /(5 K+1)$ cannot be improved, since $|a|$ could be chosen so close to $1^{-}$.

Proof of Theorem 2. Just adopt the method of proof of Theorem 1. The desired conclusion follows if we consider (2) and replace the first term $\left|a_{0}\right|$ in (2) by $\left|a_{0}\right|^{2}$. So we omit the details.

Proof of Theorem 3. We recall that if $p(z)=\sum_{n=0}^{\infty} p_{n} z^{n}$ is analytic in $\mathbb{D}$ such that $\operatorname{Re} p(z)>0$ in $\mathbb{D}$, then $\left|p_{n}\right| \leq 2 \operatorname{Re} p_{0}$ for all $n \geq 1$. Applying this result to $p(z)=1-f(z)$ leads to $\left|a_{n}\right| \leq 2\left(1-a_{0}\right)$ for all $n \geq 1$. Thus, as in the proof of Theorem 1, we can easily obtain from Lemma 1 that

$$
\sum_{n=1}^{\infty}\left|b_{n}\right|^{2} r^{n} \leq k^{2} \sum_{n=1}^{\infty}\left|a_{n}\right|^{2} r^{n} \leq 4 k^{2}\left(1-a_{0}\right)^{2} \frac{r}{1-r}
$$

and

$$
\sum_{n=1}^{\infty}\left|b_{n}\right| r^{n} \leq 2 k\left(1-a_{0}\right) \frac{r}{1-r}
$$

so that

$$
\sum_{n=0}^{\infty}\left|a_{n}\right| r^{n}+\sum_{n=1}^{\infty}\left|b_{n}\right| r^{n} \leq a_{0}+2(1+k)\left(1-a_{0}\right) \frac{r}{1-r}
$$

which is clearly less than or equal to 1 for $r \leq 1 /(3+2 k)$. Again, substituting $k=$ $(K-1) /(K+1)$ gives the desired result. Moreover, sharpness can be seen by considering functions of the form

$$
h(z)=\frac{a-z}{1-a z}, 0<a<1, \text { and } g(z)=k h(z)=\frac{K-1}{K+1} h(z) .
$$

The proof is complete.

For the proof of Theorem 4, without loss of generality, we may assume that

$$
\max \left\{\|h\|_{\infty},\|g\|_{\infty}\right\}=1 \text {. }
$$

Then, $\|h\|_{\infty} \leq 1$ and it follows from the classical Schwarz inequality that

$$
\sum_{n=1}^{\infty}\left|a_{n}\right| r^{n} \leq \sqrt{\sum_{n=1}^{\infty}\left|a_{n}\right|^{2}} \sqrt{\sum_{n=1}^{\infty} r^{2 n}} \leq \frac{r}{\sqrt{1-r^{2}}} .
$$

Again, since $\|g\|_{\infty} \leq 1$, the same inequality is valid for $b_{n}$. Thus, by combining the resulting inequality with the last inequality, we find that

$$
\sum_{n=1}^{\infty}\left(\left|a_{n}\right|+\left|b_{n}\right|\right) r^{n} \leq \frac{2 r}{\sqrt{1-r^{2}}} \leq 1 \text { for } r \leq \frac{1}{\sqrt{5}} .
$$

Although this simple approach gives a good estimate, the number $1 / \sqrt{5}$ is not sharp. In order to obtain the sharp estimate we will use a recent approach of Kayumov and Ponnusamy [11, 12] which, in particular, settled the problem Ali et al. 2] on the Bohr radius for odd analytic functions. 
Lemma 2. Suppose $p$ is a natural number and $2 r^{2 p}<1$. If $h(z)=\sum_{n=0}^{\infty} a_{p n+1} z^{p n+1}$ is analytic and $|h(z)| \leq 1$ for $z \in \mathbb{D}$, then the following inequalities hold:

$$
\sum_{n=0}^{\infty}\left|a_{p n+1}\right| r^{p n+1} \leq\left\{\begin{aligned}
\frac{1}{r^{p-1}}\left(3-2 \sqrt{2} \sqrt{1-r^{2 p}}\right) & \text { for }\left|a_{1}\right| \geq r^{p} \\
2 r^{p+1} & \text { for }\left|a_{1}\right|<r^{p} .
\end{aligned}\right.
$$

If $r^{p} \leq 1 / 3$, then always

$$
\sum_{n=0}^{\infty}\left|a_{p n+1}\right| r^{p n+1} \leq \max \left\{2 r^{p+1}, r\right\}
$$

holds.

Proof. This is a special case of the proof of Theorem 1 in [11,12]. So, we omit the details to avoid repetition.

Proof of Theorem 4. Since $3-2 \sqrt{2} \sqrt{1-r^{2}}=1 / 2$ gives $r=\sqrt{7 / 32}$ and $2 r^{2}=14 / 32<$ $1 / 2$, it follows from Lemma 2 that

$$
\sum_{n=0}^{\infty}\left|a_{n+1}\right| r^{n+1} \leq \frac{1}{2} \text { for } r \leq \sqrt{\frac{7}{32}}
$$

and the same inequality is valid for the coefficients $b_{n}$ :

$$
\sum_{n=0}^{\infty}\left|b_{n+1}\right| r^{n+1} \leq \frac{1}{2} \text { for } r \leq \sqrt{\frac{7}{32}}
$$

Summing these two inequalities immediately proves that

$$
\sum_{n=1}^{\infty}\left(\left|a_{n}\right|+\left|b_{n}\right|\right) r^{n} \leq 1 \text { for } r \leq \sqrt{\frac{7}{32}} .
$$

To prove that the number $\sqrt{7 / 32}$ cannot be replaced by a larger one, we let $a=3 / \sqrt{14}$, $\lambda \in \partial \mathbb{D}$ and consider

$$
h(z)=z\left(\frac{a-z}{1-a z}\right)=a z-\left(1-a^{2}\right) z^{2} \sum_{n=2}^{\infty}(a z)^{n-2} \text { and } g(z)=\lambda h(z) .
$$

Then, we find that

$$
\sum_{n=1}^{\infty}\left(\left|a_{n}\right|+\left|b_{n}\right|\right) r^{n}=2\left(a r+\frac{\left(1-a^{2}\right) r^{2}}{1-a r}\right)=1 \text { for } r=\sqrt{\frac{7}{32}}
$$

which shows that the number $\sqrt{7 / 32}$ cannot be improved.

Proof of Theorem 5. Let $r=1 / 2$. Since $r^{p}<1 / 3$ for $p \geq 2$, it follows from Lemma 2 and the hypothesis that

$$
\sum_{n=0}^{\infty}\left|a_{p n+1}\right| r^{p n+1} \leq \frac{1}{2} \text { for } r \leq \frac{1}{2}
$$


and the same inequality is valid for the coefficients $b_{n}$. As a consequence of adding these two inequalities, we obtain that

$$
\sum_{n=0}^{\infty}\left(\left|a_{p n+1}\right|+\left|b_{p n+1}\right|\right) r^{p n+1} \leq 1 \text { for } r \leq \frac{1}{2} .
$$

The function $f(z)=z+\bar{z}$ shows that $1 / 2$ is sharp.

\section{FurTher RESUlTS ON BOHR RADIUS FOR QUASICONFORMAL HARMONIC MAPPINGS}

Theorem 6. Suppose that $f(z)=h(z)+\overline{g(z)}=\sum_{n=0}^{\infty} a_{n} z^{n}+\overline{\sum_{n=2}^{\infty} b_{n} z^{n}}$ is a sensepreserving $K$-quasiconformal harmonic mapping of the disk $\mathbb{D}$, where $h$ is a bounded function in $\mathbb{D}$. Then

$$
\sum_{n=0}^{\infty}\left|a_{n}\right| r^{n}+\sum_{n=2}^{\infty}\left|b_{n}\right| r^{n} \leq\|h\|_{\infty} \text { for } r \leq r_{K}
$$

where $r_{K}$ is the positive root of the equation $M_{K}(r)=1 / 2$ and

$$
M_{K}(r)=\frac{r}{1-r}+\left(\frac{K-1}{K+1}\right) r^{2} \sqrt{\frac{1+r^{2}}{\left(1-r^{2}\right)^{3}}} \sqrt{\frac{\pi^{2}}{6}-1} .
$$

The number $r_{K}$ cannot be replaced by a number greater than $R=R(K)$, where $R$ is the positive root of the equation

$$
\frac{4 R}{1-R}\left(\frac{K}{K+1}\right)+2\left(\frac{K-1}{K+1}\right) \log (1-R)=1
$$

Proof. Without loss of generality we may assume that $\|h\|_{\infty} \leq 1$. Because $f=h+\bar{g}$ is sense-preserving and $K$-quasiconformal harmonic mapping with $g^{\prime}(0)=0$, Schwarz's lemma gives that $\omega_{f}=: \omega=g^{\prime} / h^{\prime}$ is analytic in $\mathbb{D}$ and $|\omega(z)| \leq k|z|$ in $\mathbb{D}$, where $k=$ $(K-1) /(K+1)$. Thus, we have

$$
\left|g^{\prime}(z)\right|^{2}=\left|\omega(z) h^{\prime}(z)\right|^{2} \leq k^{2}\left|z h^{\prime}(z)\right|^{2} .
$$

We integrate this inequality over the circle $|z|=r$ and obtain

$$
\sum_{n=2}^{\infty} n^{2}\left|b_{n}\right|^{2} r^{2(n-1)} \leq k^{2} r^{2} \sum_{n=1}^{\infty} n^{2}\left|a_{n}\right|^{2} r^{2(n-1)}
$$

and, because $\left|a_{n}\right| \leq 1-\left|a_{0}\right|^{2}$ for $n \geq 1$, we have

$$
\sum_{n=2}^{\infty} n^{2}\left|b_{n}\right|^{2} r^{2 n} \leq k^{2}\left(1-\left|a_{0}\right|^{2}\right)^{2} r^{4} \sum_{n=1}^{\infty} n^{2} r^{2(n-1)}=k^{2}\left(1-\left|a_{0}\right|^{2}\right)^{2} \frac{r^{4}\left(1+r^{2}\right)}{\left(1-r^{2}\right)^{3}} .
$$

Consequently, using the classical Schwarz inequality, we deduce that

$$
\sum_{n=2}^{\infty}\left|b_{n}\right| r^{n} \leq \sqrt{\sum_{n=2}^{\infty} n^{2}\left|b_{n}\right|^{2} r^{2 n}} \sqrt{\sum_{n=2}^{\infty} \frac{1}{n^{2}}} \leq k r^{2}\left(1-\left|a_{0}\right|^{2}\right) \sqrt{\frac{1+r^{2}}{\left(1-r^{2}\right)^{3}}} \sqrt{\frac{\pi^{2}}{6}-1} .
$$


Therefore, from (8) it follows that

$$
\begin{aligned}
S & =\left|a_{0}\right|+\left|a_{1}\right| r+\sum_{n=2}^{\infty}\left(\left|a_{n}\right|+\left|b_{n}\right|\right) r^{n} \\
& \leq\left|a_{0}\right|+\left(1-\left|a_{0}\right|^{2}\right)\left[\frac{r}{1-r}+k r^{2} \sqrt{\frac{1+r^{2}}{\left(1-r^{2}\right)^{3}}} \sqrt{\frac{\pi^{2}}{6}-1}\right]
\end{aligned}
$$

which is less than or equal to 1 provided $M_{K}(r)=1 / 2$ holds, where $M_{K}(r)$ is defined by (6).

Finally, we let $a \in[0,1)$ and consider the functions

$$
h(z)=\frac{a-z}{1-a z} \text { and } g^{\prime}(z)=k z h^{\prime}(z) .
$$

From here we find that

$$
\left|a_{n}\right|=a^{n-1}\left(1-a^{2}\right) \text { and }\left|b_{n}\right|=k\left(\frac{n-1}{n}\right) a^{n-2}\left(1-a^{2}\right), \quad n \geq 2,
$$

so that

$$
\begin{aligned}
\sum_{n=0}^{\infty}\left|a_{n}\right| r^{n}+\sum_{n=2}^{\infty}\left|b_{n}\right| r^{n} & =a+\left(1-a^{2}\right) \sum_{n=1}^{\infty} a^{n-1} r^{n}+k\left(1-a^{2}\right) \sum_{n=2}^{\infty} \frac{n-1}{n} a^{n-2} r^{n} \\
& =a+\left(1-a^{2}\right) \frac{r}{1-a r}+k\left(1-a^{2}\right) \frac{a r+(1-a r) \log (1-a r)}{a^{2}(1-a r)} \\
& =a+\left(1-a^{2}\right)\left[\frac{(a+k) r}{a(1-a r)}+k \frac{\log (1-a r)}{a^{2}}\right],
\end{aligned}
$$

where $k=(K-1) /(K+1)$. Simple analysis shows that the last expression is less than or equal to 1 for all $a \in[0,1)$ only in the case when $r \leq R=R(K)$ which is the positive root of the equation (7).

Allowing $K \rightarrow \infty$ in Theorem [6 shows that the root $r_{\infty}$ of the limiting case $M_{\infty}(r)=$ $1 / 2$, i.e.,

$$
\frac{r}{1-r}+r^{2} \sqrt{\frac{1+r^{2}}{\left(1-r^{2}\right)^{3}}} \sqrt{\frac{\pi^{2}}{6}-1}=\frac{1}{2},
$$

gives the value $0.2942 \ldots$. We may now formulate this discussion as follows.

Corollary 3. Suppose that $f(z)=h(z)+\overline{g(z)}=\sum_{n=0}^{\infty} a_{n} z^{n}+\overline{\sum_{n=2}^{\infty} b_{n} z^{n}}$ is a sensepreserving harmonic mapping of the disk $\mathbb{D}$, where $h$ is a bounded function in $\mathbb{D}$. Then

$$
\sum_{n=0}^{\infty}\left|a_{n}\right| r^{n}+\sum_{n=2}^{\infty}\left|b_{n}\right| r^{n} \leq\|h\|_{\infty} \text { for } r \leq 0.2942 \ldots
$$

The number $0.2942 \ldots$ cannot be replaced by a number greater than $R=0.299825 \ldots$, where $R$ is the positive root of the equation

$$
\frac{4 R}{1-R}+2 \log (1-R)=1 .
$$

Further remarks would be useful. 
Remark 1. Also, it is worth pointing out that if the first term $\left|a_{0}\right|$ in (5) is replaced by $\left|a_{0}\right|^{2}$, then the conclusion of Corollary 3 takes the form

$$
\left|a_{0}\right|^{2}+\sum_{n=1}^{\infty}\left|a_{n}\right| r^{n}+\sum_{n=2}^{\infty}\left|b_{n}\right| r^{n} \leq\|h\|_{\infty} \text { for } r \leq r_{K}
$$

where $r_{K}$ is the positive root of the equation $M_{K}(r)=1$ and $M_{K}(r)$ is defined by (6). The number $r_{K}$ cannot be replaced by a number greater than $R=R(K)$, where $R$ is the positive root of the equation

$$
\frac{2 R}{1-R}\left(\frac{K}{K+1}\right)+\left(\frac{K-1}{K+1}\right) \log (1-R)=1 .
$$

Again, the case $K \rightarrow \infty$ (i.e. when the dilatation has the property that $|\omega(z)|<1$ in $\mathbb{D})$ needs a special mention, since the corresponding value of $r_{\infty}$ is $0.435668 \ldots$, where the number $0.435668 \ldots$ is the root of the equation $M_{\infty}(r)=1$, i.e.,

$$
\frac{r}{1-r}+r^{2} \sqrt{\frac{1+r^{2}}{\left(1-r^{2}\right)^{3}}} \sqrt{\frac{\pi^{2}}{6}-1}=1 .
$$

Furthermore, the number $0.435668 \ldots$ cannot be replaced by a number greater than $R(\infty)=$ $0.44182 \ldots$, where $R(\infty)$ is the positive root of the equation

$$
\frac{2 R}{1-R}+\log (1-R)=1 \text {. }
$$

Theorem 7. Suppose that $f(z)=h(z)+\overline{g(z)}=\sum_{n=0}^{\infty} a_{n} z^{n}+\overline{\sum_{n=0}^{\infty} b_{n} z^{n}}$ is a locally univalent $K$-quasiconformal harmonic mapping of the disk $\mathbb{D}$, where $h^{\prime}$ is a bounded function in $\mathbb{D}$. Then

$$
\frac{K+1}{2 K} \sum_{n=1}^{\infty}\left(\left|a_{n}\right|+\left|b_{n}\right|\right) n r^{n-1} \leq\left\|h^{\prime}\right\|_{\infty} \text { for } r \leq 1 / 3
$$

and the number $1 / 3$ is sharp.

The proof of Theorem 7 easily follows easily if we use the above method and the classical proof of Bohr's 1/3-Theorem. The case $K \rightarrow \infty$ gives the corresponding result for sense-preserving harmonic mappings of the disk $\mathbb{D}$.

\section{BOHR RADIUS FOR HARMONIC BLOCH FUNCTIONS}

A harmonic function $f$ is called a harmonic Bloch function if and only if

$$
\sup _{z \in \mathbb{D}}\left(1-|z|^{2}\right) \Lambda_{f}(z)<+\infty
$$

where $\Lambda_{f}(z)=\left|f_{z}(z)\right|+\left|f_{\bar{z}}(z)\right|$. The space of all harmonic Bloch functions, denoted by the symbol $\mathcal{B}_{H}$, forms a complex Banach space with the norm $\|\cdot\|$ given by (see [9])

$$
\|f\|_{\mathcal{B}_{H}}=|f(0)|+\sup _{z \in \mathbb{D}}\left(1-|z|^{2}\right) \Lambda_{f}(z)
$$

where $\Lambda_{f}(z)=\left|f_{z}(z)\right|+\left|f_{\bar{z}}(z)\right|$. This is referred to as the harmonic Bloch norm and the elements of the harmonic Bloch space are called harmonic Bloch functions. Recently, the 
space $\mathcal{B}_{H}$ together with its various generalizations have been studied extensively. See for example, see [6] 8 ].

Clearly, this definition coincides with the classical Bloch space $\mathcal{B}$ when $f$ is analytic in $\mathbb{D}$. We refer to the basic paper on this topic by Anderson et al. [3] and the book of Pommerenke [15].

Theorem 8. Let $f \in \mathcal{B}$ and $\|f\|_{\mathcal{B}} \leq 1$. Then

$$
\sum_{n=0}^{\infty}\left|a_{n}\right| r^{n} \leq 1 \text { for } r \leq R=0.55356 \ldots
$$

where $R$ is the positive solution to the equation

$$
1-R+R \log (1-R)=0 .
$$

The number $R$ cannot be replaced by a number greater than $0.624162 \ldots$.

Proof. Let $f(z)=\sum_{n=0}^{\infty} a_{n} z^{n}$ be analytic and

$$
\|f\|_{\mathcal{B}}=|f(0)|+\sup _{z \in \mathbb{D}}\left(1-|z|^{2}\right)\left|f^{\prime}(z)\right| \leq 1 .
$$

Thus, we have

$$
\left|f^{\prime}(z)\right|^{2} \leq \frac{\left(1-\left|a_{0}\right|\right)^{2}}{\left(1-|z|^{2}\right)^{2}}
$$

We integrate this inequality over the circle $|z|=r$ and obtain

$$
\sum_{n=1}^{\infty} n^{2}\left|a_{n}\right|^{2} r^{2 n} \leq r^{2} \frac{\left(1-\left|a_{0}\right|\right)^{2}}{\left(1-r^{2}\right)^{2}}
$$

so that

$$
\sum_{n=1}^{\infty} n^{2}\left|a_{n}\right|^{2} r^{n-1} \leq \frac{\left(1-\left|a_{0}\right|\right)^{2}}{(1-r)^{2}} \text { for } r<1 .
$$

We may now integrate this with respect to $r$ (with limit from 0 to $r$ ) and obtain

$$
\sum_{n=1}^{\infty} n\left|a_{n}\right|^{2} r^{n-1} \leq \frac{\left(1-\left|a_{0}\right|\right)^{2}}{1-r} \text { for } r<1
$$

which by integration with respect to $r$ gives

$$
\sum_{n=1}^{\infty}\left|a_{n}\right|^{2} r^{n} \leq\left(1-\left|a_{0}\right|\right)^{2} \log \frac{1}{1-r} \text { for } r<1 .
$$

Consequently,

$$
\sum_{n=1}^{\infty}\left|a_{n}\right| r^{n} \leq \sqrt{\sum_{n=1}^{\infty}\left|a_{n}\right|^{2} r^{n}} \sqrt{\sum_{n=1}^{\infty} r^{n}} \leq\left(1-\left|a_{0}\right|\right) \sqrt{\log \frac{1}{1-r}} \sqrt{\frac{r}{1-r}} .
$$

It means that

$$
\sum_{n=0}^{\infty}\left|a_{n}\right| r^{n} \leq\left|a_{0}\right|+\left(1-\left|a_{0}\right|\right) \sqrt{\log \frac{1}{1-r}} \sqrt{\frac{r}{1-r}} \leq 1 \text { for } r \leq R,
$$


where $R=0.55356 \ldots$ is the positive solution to the equation

$$
\log \frac{1}{1-R}=\frac{1-R}{R} .
$$

Now we consider (see [4])

$$
f(z)=\frac{3 \sqrt{3}}{4}\left(\left(\frac{z-a}{1-a z}\right)^{2}-a^{2}\right)=\frac{3 \sqrt{3}}{4}\left(\sum_{n=1}^{\infty} a_{n} z^{n}\right),
$$

where $a \in(0,1), a_{1}=-2 a\left(1-a^{2}\right)$ and

$$
a_{n}=\left(1-a^{2}\right)\left[n\left(1-a^{2}\right)-\left(1+a^{2}\right)\right](a)^{n-2} \text { for } n \geq 2 .
$$

Since

$$
f^{\prime}(z)=\frac{3 \sqrt{3}}{2}\left(\frac{z-a}{1-a z}\right) \frac{1-a^{2}}{(1-a z)^{2}},
$$

it is easy to check that $\|f\|_{\mathcal{B}}=1$. For this function, we observe that the coefficients $a_{n}$ for $n \geq 2$ are all positive, whenever $a \in(0,1 / \sqrt{3})$. Furthermore,

$$
\sum_{n=0}^{\infty}\left|a_{n}\right| r^{n}=\frac{3 \sqrt{3}}{4}\left(\left(\frac{r-a}{1-a r}\right)^{2}-a^{2}+4 a\left(1-a^{2}\right) r\right) .
$$

Now we suppose that $r$ is a function of $a$. We want to find the minimal $r$ for which

$$
\frac{3 \sqrt{3}}{4}\left(\left(\frac{r-a}{1-a r}\right)^{2}-a^{2}+4 a\left(1-a^{2}\right) r\right)=1
$$

which may be rewritten as

$$
(r-a)^{2}+(1-r a)^{2}\left(-a^{2}+4 a\left(1-a^{2}\right) r\right)=\frac{4}{3 \sqrt{3}}(1-a r)^{2} .
$$

Now we differentiate $r$ in the variable $a$ and then we set $r^{\prime}(a)=0$. We arrive at the cubic equation

$$
18 r+8 \sqrt{3} r-54 a^{2} r-144 a r^{2}-8 \sqrt{3} a r^{2}+252 a^{3} r^{2}+108 a^{2} r^{3}-180 a^{4} r^{3}=0 .
$$

The algebraic system of the equations (10) and (11) can be easily solved, for example, by Mathematica 10. Consequently, we get $a=0.3775$ and then we obtain $r=0.624162$ such that $\sum_{n=0}^{\infty}\left|a_{n}\right| r^{n}=1$. The proof is complete.

Theorem 9. Suppose that $f=h+\bar{g}$ is harmonic in $\mathbb{D}, g(0)=0$ and $\|f\|_{\mathcal{B}_{H}} \leq 1$, where

$$
\|f\|_{\mathcal{B}_{H}}=|f(0)|+\sup _{z \in \mathbb{D}}\left(1-|z|^{2}\right)\left(\left|h^{\prime}(z)\right|+\left|g^{\prime}(z)\right|\right) .
$$

Then

$$
\left|a_{0}\right|+\sum_{n=1}^{\infty} \sqrt{\left|a_{n}\right|^{2}+\left|b_{n}\right|^{2}} r^{n} \leq 1 \text { for } r \leq R=0.55356 .
$$

This number 0.55356 cannot be replaced by a number greater than $0.624162 \ldots$. 
Proof. By assumption, we have

$$
\left|h^{\prime}(z)\right|^{2}+\left|g^{\prime}(z)\right|^{2} \leq \frac{\left(1-\left|a_{0}\right|\right)^{2}}{\left(1-|z|^{2}\right)^{2}} .
$$

We integrate this inequality over the circle $|z|=r$ and obtain

$$
\sum_{n=1}^{\infty} n^{2}\left(\left|a_{n}\right|^{2}+\left|b_{n}\right|^{2}\right) r^{2 n} \leq r^{2} \frac{\left(1-\left|a_{0}\right|\right)^{2}}{\left(1-r^{2}\right)^{2}} .
$$

Now the remaining part of the proof is identical to that of Theorem 8 . Thus, the proof is complete.

As remarked earlier if we replace the first term $\left|a_{0}\right|$ in the conclusion of the last two theorems by $\left|a_{0}\right|^{2}$, then the Bohr radius obviously can be stated in an improved form.

\section{Conclusion}

We conclude the paper with the following conjectures.

Conjecture 1. Suppose that $f=h+\bar{g}=\sum_{n=1}^{\infty} a_{n} z^{n}+\overline{\sum_{n=1}^{\infty} b_{n} z^{n}}$ is a harmonic mapping of the disk $\mathbb{D}$, where $h$ is bounded in $\mathbb{D}$. If $\left|g^{\prime}(z)\right| \leq \frac{K-1}{K+1}\left|h^{\prime}(z)\right|$, then

$$
\sum_{n=1}^{\infty}\left(\left|a_{n}\right|+\left|b_{n}\right|\right) r^{n} \leq\|h\|_{\infty} \text { for } r \leq \frac{1}{4} \sqrt{\frac{7}{2}-\frac{1}{2 K^{2}}+\frac{5}{K}}
$$

This constant is sharp for all $K \geq 1$.

The conjectured extremal function has the form

$$
f(z)=h(z)+\frac{K-1}{K+1} \overline{h(z)}, \quad h(z)=z\left(\frac{z-a}{1-\bar{a} z}\right),
$$

with a suitable $a \in \mathbb{D}$.

If we replace the condition " $\left|g^{\prime}(z)\right| \leq \frac{K-1}{K+1}\left|h^{\prime}(z)\right|$ " by " $f$ is $K$-quasiconformal", then the Bohr radius will be greater than the number mentioned in Conjecture 1, because the conjectured extremal function is not locally univalent in the unit disk.

The proof of Theorem 5 leads to another problem which we state it now as a conjecture.

Conjecture 2. Let $p \geq 2$. Suppose that $f(z)=h(z)+\overline{g(z)}=\sum_{n=0}^{\infty} a_{n} z^{p n+1}+\overline{\sum_{n=0}^{\infty} b_{n} z^{p n+1}}$ is a harmonic p-symmetric and sense-preserving mapping in $\mathbb{D}$, where $h$ and $g$ are bounded functions in $\mathbb{D}$. Then

$$
\sum_{n=0}^{\infty}\left(\left|a_{n}\right|+\left|b_{n}\right|\right) r^{p n+1} \leq \max \left\{\|h\|_{\infty},\|g\|_{\infty}\right\} \text { for } r \leq \frac{1}{2} .
$$

The constant $1 / 2$ is sharp.

Also it would be interesting to obtain an analog of the Conjecture 2 for locally $K$ quasiconformal mappings. 
Acknowledgements. The research of the first author was supported by Russian foundation for basic research, Proj. 17-01-00282, and the research of the second author was supported by the project RUS/RFBR/P-163 under Department of Science \& Technology (India). The second author is currently on leave from the IIT Madras.

\section{REFERENCES}

1. Y. Abu-Muhanna, R. M. Ali, and S. Ponnusamy, On the Bohr inequality, In "Progress in Approximation Theory and Applicable Complex Analysis" (Edited by N.K. Govil et al. ), Springer Optimization and Its Applications 117 (2016), 265-295.

2. R. M. Ali, R. W. Barnard and A. Yu. Solynin, A note on the Bohr's phenomenon for power series, J. Math. Anal. Appl. 449(1) (2017), 154-167.

3. J. M. Anderson, J. G. Clunie and Ch. Pommerenke, On Bloch functions and normal functions, J. Reine Angew. Math. 270 (1974), 12-37.

4. F. G. Avkhadiev and I. R. Kayumov, Estimates for Bloch functions and their generalization, Doklady Akademii Nauk 349(5) (1996), 583-585.

5. H. Bohr, A theorem concerning power series, Proc. London Math. Soc. 13(2) (1914), 1-5.

6. Sh. Chen, S. Ponnusamy and X. Wang, Landaus theorem and Marden constant for harmonic $\nu$-bloch mappings, Bull. Aust. Math. Soc. 84 (2011), 19-32.

7. Sh. Chen, S. Ponnusamy and X. Wang, Coefficient estimates and Landau-Bloch's theorem for planar harmonic mappings, Bull. Malaysian Math. Sciences Soc. 34(2) (2011), 255-265.

8. Sh. Chen, S. Ponnusamy and X. Wang, Landau-Bloch constants for functions in $\alpha$-Bloch spaces and Hardy spaces, Complex Anal. Oper. Theory 6 (2012), 1025-1036.

9. F. Colonna, The Bloch constant of bounded harmonic mappings, Indiana Univ. Math. J. 38(1989), 829-840.

10. D. Kalaj and M. Vuorinen, On harmonic functions and the Schwarz lemma, Proc. Amer. Math. Soc., 140(1) (2012), 161-165.

11. I. R. Kayumov and S. Ponnusamy, Bohr inequality for odd analytic functions, Comput. Methods Funct. Theory, (2017) Available online; DOI 10.1007/s40315-017-0206-2

See also https://arxiv.org/pdf/1701.03884.pdf

12. I. R. Kayumov and S. Ponnusamy, Bohr's inequality for analytic functions $\sum_{k} b_{k} z^{k p+m}$ and harmonic functions, See https://arxiv.org/abs/1708.05578

13. O. Lehto and K. I. Virtanen, Quasiconformal mappings in the plane, Springer Verlag, 1973.

14. H. Lewy, On the non-vanishing of the Jacobian in certain one-to-one mappings, Bull. Amer. Math. Soc., 42 (1936), 689-692.

15. Ch. Pommerenke, Boundary behaviour of conformal maps, Springer, New York, 1992.

16. M. Vuorinen, Conformal geometry and quasiregular mappings, Lecture Notes in Math. Vol. 1319, Springer-Verlag, 1988.

I. R Kayumov, Kazan Federal University, Kremlevskaya 18, 420008 Kazan, Russia

E-mail address: ikayumov@kpfu.ru

S. Ponnusamy, Stat-Math Unit, Indian Statistical Institute (ISI), Chennai Centre, 110 ,

Nelson Manickam Road, Aminjikarai, Chennai, 600 029, India.

E-mail address: samy@isichennai.res.in, samy@iitm.ac.in

N. Shakirov, Kazan Federal University, Kremlevskaya 18, 420008 Kazan, Russia 